ORIGINAL ARTICLE

\title{
Immunohistochemical and molecular assessment of human herpesvirus type 8 in gastrointestinal tumours
}

\author{
C C Su, C F Li, Y L Liao, C N Lin, J J Lu
}

J Clin Pathol 2005;58:856-859. doi: 10.1136/icp.2004.023622 See end of article for
authors' affiliations
$\ldots \ldots \ldots \ldots \ldots \ldots \ldots \ldots \ldots$
Correspondence to:
Dr C-C Su, Department of
Anatomic Pathology,
Buddhist Dalin Tzu Chi
General Hospital, 2,
Minsheng Road, Dalin
Town, Chiayi County 622,
Taiwan; sucpo@yahoo.
com.tw
Accepted for publication
23 February 2005
Background: Gastrointestinal stromal tumours (GISTs) are the most common mesenchymal tumours of the gastrointestinal tract, histologically identified as highly cellular spindle or epithelioid cell tumours, and often positive for CD34 (60-70\%). Kaposi's sarcomas (KSs) are similar to GISTs: they are most often found in the gastrointestinal tract (although cutaneous lesions do occur), they are also composed of spindle or epithelioid cells (although erythrocytes are also seen), and the tumour cells are nearly all positive for CD34. Human herpesvirus type $8(\mathrm{HHV}-8)$ DNA has been found consistently in all types of KS, in particular in CD34 positive KS tumour cells. However, the association between HHV-8 and GIST has not been investigated.

Aims: To assess the presence of HHV-8 in GISTs.

Methods: Paraffin wax embedded tissues of 86 primary GISTs and their recurrent or metastatic tumours were analysed immunohistochemically for the CD34 antigen and HHV-8 latent nuclear antigen 1 (LNA-1) and by means of the nested polymerase chain reaction (PCR) and real time PCR for HHV-8 DNA.

Results: None of the 86 GISTs contained HHV-8 DNA sequences or LNA-1 positive cells.

Conclusions: These results demonstrate the lack of HHV-8 infection in GIST tumour cells. HHV-8 does not appear to play a role in the pathogenesis of GIST, irrespective of the status of the tumour.
G astrointestinal stromal tumours (GISTs) are the most common mesenchymal tumours of the gastrointestinal tract, ${ }^{1}$ with most being found in the stomach $(60$ $70 \%$ ) and small intestine (25-35\%). ${ }^{2}$ They are histologically identified as highly cellular spindle or epithelioid cell tumours. These tumours often express c-kit (CDl17 antigen), which is a major diagnostic criterion. ${ }^{3}$ Additional features include positivity for CD34 (60-70\% of tumours), and variable expression of smooth muscle actins (20-30\%) and S100 protein $(10 \%){ }^{45}$

\begin{abstract}
"Human herpesvirus type 8 DNA has been found consistently in all types of Kaposi's sarcoma and is always present in the CD34 positive spindle or epithelioid tumour cells of this tumour"
\end{abstract}

Kaposi's sarcomas (KSs) are similar to GISTs in that they are most often found in the gastrointestinal tract (although cutaneous lesions do occur), ${ }^{6}$ they are also composed of spindle or epithelioid cells (although erythrocytes are also seen), ${ }^{7}$ and the tumour cells are nearly all positive for the CD34 antigen. ${ }^{8} 9$ To date, human herpesvirus type 8 (HHV-8) DNA has been found consistently in all types of $\mathrm{KS},{ }^{10}{ }^{11}$ and is always present in the CD34 positive spindle or epithelioid tumour cells of KS. ${ }^{78}$ In addition, it has also been reported in the CD34 positive spindle cells of other lesions, such as the subcapsular cells in Castleman's disease, ${ }^{12}$ the spindle cells in angioproliferative disorder, ${ }^{13}$ and the spindle cells in pyogenic granuloma. ${ }^{14}$ Although the absence of Epstein-Barr virus has been reported in GIST, ${ }^{15}$ the relation between GIST and other viruses, in particular HHV-8, has not been described previously.

Our present retrospective study was performed to assess the presence of HHV-8 in GISTs.

\section{MATERIALS AND METHODS Clinicopathological data}

The pathological files of the Chi Mei Medical Centre, Taiwan from 1992 to 2002 were searched for GIST, leiomyomas, leiomyosarcomas, leiomyoblastomas, smooth muscle tumours, neurilemmomas, neurofibromas, malignant peripheral nerve sheath tumours, spindle cell tumours, and mesenchymal tumours in the gastrointestinal tract. Only patients with primary surgical intervention in this hospital were included in our study. Those with recurrent or metastatic tumours after previous surgical manipulation in other institutes were excluded.

Clinical data were reviewed, including sex, age, tumour location, tumour size, follow up duration, the interval between initial diagnosis and malignant presentation, and the status of malignancy.

\section{Immunohistochemical studies}

Immunostaining for CD34, CD117, and HHV-8 latent nuclear antigen 1 (LNA-1) was performed using the labelled streptavidin-biotin peroxidase method (LSAB kit; Dako Corporation, Carpinteria, California, USA). Formalin fixed, paraffin wax embedded tissue blocks were cut at $5 \mu \mathrm{m}$, dried overnight at $60^{\circ} \mathrm{C}$, and dewaxed in xylene. Subsequently, sections were rehydrated through graded alcohols to water. Heat induced epitope retrieval was achieved by boiling sections in 0.01M citrate buffer (Euroclone, West York, UK) at $\mathrm{pH} 6.0$ in a microwave oven at $900 \mathrm{~W}$ for 12.5 minutes. Sections were then cooled in running water for 20 minutes, rinsed thoroughly, and placed in phosphate buffered saline (PBS) for one minute, three times. Endogenous peroxidase was blocked with SuperBlock ${ }^{\circledR}$ blocking buffer (Pierce, Rockford, Illinois, USA) in PBS for 10 minutes, and slides were rinsed and washed with PBS. The following primary antibodies were added: anti-CD34 (clone QBEND 10; 1/50 dilution for 30 minutes; Serotec Corporation, Oxford, UK), anti-CD117 (1/300 dilution for 30 minutes; Dako), and a

Abbreviations: GIST, gastrointestinal tumour; $\mathrm{HHV}-8$, human herpesvirus type 8; HPF, high power field; KS, Kaposi's sarcoma; LNA1, latent nuclear antigen 1; PBS, phosphate buffered saline; PCR, polymerase chain reaction 
mouse monoclonal antibody to HHV-8 LNA-1 (NCL-HHV8LNA; clone 13B10; 1/25 dilution for 60 minutes; Novocastra Laboratories, Newcastle upon Tyne, UK). Immunostaining was performed with an OptiMax plus automated cell stainer (BioGenex, San Rammon, California, USA).

The immunohistochemistry results and the histological diagnoses were confirmed by three surgical pathologists. In total, 115 primary gastrointestinal mesenchymal tumours were identified; only those with diffuse cytoplasmic reactivity for CD117 were diagnosed as GISTs and 86 cases were used for our study. Cytoplasmic or cytoplasmic membrane staining of more than $10 \%$ of tumour cells was considered positive for CD34. For HHV-8 LNA-1, only nuclear staining was considered positive.

\section{DNA extraction}

Paraffin wax sections of the GIST samples were used for our study. DNA was prepared by means of a QIAamp tissue kit (Qiagen, Hilden, Germany). Briefly, $1.2 \mathrm{ml}$ xylene was added to the paraffin wax embedded tissues (two $5 \mu \mathrm{m}$ sections), which were then washed with ethanol. Next, genomic DNA of wax embedded tissues was obtained according to the QIAamp protocol. Only sections containing at least $85 \%$ tumour cells (no more than 15\% additional non-tumour material) were used for DNA extraction.

\section{Primers and probes}

Primers and probes were used according to the method reported by Muller and colleagues ${ }^{16}$ and Stamey et al. ${ }^{17}$ For the nested PCR assay, the outer primer pair KSI (5'-AGC CGA AAG GAT TCC ACC AT- $3^{\prime}$ ) and KS2 (5'-TCC GTG TTG TCT ACG TCC AG-3') was used to produce a 233 bp DNA fragment; the inner primer pair NSI $\left(5^{\prime}\right.$-ACG GAT TTG ACC CCG TGT TC-3') and NS2 (5'-AAT GAC ACA TTG GTG GTA TA-3') was used to produce a 160 bp DNA fragment. For the real time PCR assay, forward (5'-CCA CCC TCG AAT GCA CAA C-3') and reverse ( $5^{\prime}$-GTC GGG ATC GGG AAA AGC T$\left.3^{\prime}\right)$ primers, the fluorogenic Taqman probe (5'-CCA CCC AGT CAG CCC AGG CAC TAA AC-3'), and PE Applied Biosystems (Primer Express software; PE Applied Biosystems, Foster City, California, USA) were applied to amplify and detect a $49 \mathrm{bp}$ amplicon in the HHV-8 minor capsid protein gene (open reading frame 25).

\section{Nested PCR}

The following conditions were used for amplification: 25 pmol of each primer, $200 \mu \mathrm{M}$ of each dNTP, $5 \mu \mathrm{l} 10 \times$ PCR buffer, 1 U Taq (Promega, Southampton, UK), 1.5mM $\mathrm{Mg}^{2+}$, and $5 \mu \mathrm{l}$ extracted DNA in a total volume of $50 \mu \mathrm{l}$. Reactions were carried out in a Perkin-Elmer thermocycler (Model 2400; Perkin-Elmer Cetus, Emeryville, California, USA). An initial denaturation step at $95^{\circ} \mathrm{C}$ for three minutes was followed by 35 cycles of $95^{\circ} \mathrm{C}$ for one minute, $58^{\circ} \mathrm{C}$ for two minutes, and $72^{\circ} \mathrm{C}$ for three minutes, with a final 10 minute extension at $72^{\circ} \mathrm{C}$. The products of the first PCR $(2 \mu \mathrm{l})$ were added to new reaction mixtures with the inner primer pair, and subjected to the same conditions. Products were run on a $1.5 \%$ agarose gel, stained with ethidium bromide, and viewed under ultraviolet light.

\section{Real time PCR}

TaqMan reagents and enzymes were obtained from PE Applied Biosystems. Each $25 \mu$ l PCR contained $1 \times$ TaqMan universal PCR master mix containing uracil- $N$-glycosylase (AmpErase), 500nM each HHV-8 primer, 100nM HHV-8 probe, $0.2 \mathrm{fg}$ of exogenous internal positive control DNA (TaqMan exogenous internal positive control), $1 \times$ TaqMan exogenous IPC primer and probe (VIC labelled probe) mix, and $5 \mu$ of template DNA. After a two minute incubation at $50^{\circ} \mathrm{C}$ for activation of the uracil- $N$-glycosylase, the Taq polymerase (Amplitaq Gold) was activated at $95^{\circ} \mathrm{C}$ for 10 minutes. Forty cycles of PCR were performed, each consisting of $95^{\circ} \mathrm{C}$ for 15 seconds and $60^{\circ} \mathrm{C}$ for one minute. Amplification was carried out in an ABI Prism 7000 sequence detection system (PE Biosystems), which permitted continuous automated reading of fluorescence intensities during PCR.

Our study was approved by the institutional review board of the Chi Mei Medical Centre.

\section{RESULTS}

\section{Clinical data}

The 86 patients were aged between 25 and 84 years (mean, 67; median, 65). The male to female ratio was 39: 47 (1: 1.2). Two patients had a previous history of colon adenocarcinoma and one of prostatic adenocarcinoma. At initial presentation, 82 patients had tumours limited to the primary site, two had intra-abdominal dissemination, one had liver metastasis, and one had regional lymph node metastasis.

Of the 86 patients, 49 had gastric tumours, 34 had small intestinal tumours, two had rectal tumours, and one had an oesophageal tumour.

\section{Macroscopic observation}

All tumours were well demarcated. The overlying mucosa was usually ulcerated, particularly when the tumours were larger than $5 \mathrm{~cm}$ in maximum diameter. The primary tumour sizes varied from 0.5 to $27 \mathrm{~cm}$ (mean, 7.8, 7.3, and 4.5 in the stomach, small bowel, and rectum, respectively). Nineteen tumours were larger than $10 \mathrm{~cm}, 40$ were between 5 and $10 \mathrm{~cm}$, and only 27 tumours were smaller than $5 \mathrm{~cm}$.

\section{Microscopic observation}

Microscopically, 82 cases were of the spindle cell type and four were mixed epithelioid and spindle cell type; there were no cases of the pure epithelioid type. The mitotic counts ranged from 1 to 33 mitoses/50 high power fields (HPF). Seventy two cases had mitotic counts of no more than $5 / 50$ HPF.

\section{Immunohistochemical studies}

The spindle cell components of all these 86 GISTs expressed CD117 diffusely and strongly; the epithelioid components of the four cases of mixed cell type showed diffuse but weaker cytoplasmic immunoreactivity for CD117. Sixty six cases expressed diffuse cytoplasmic staining for CD34. Only 14 of the 34 small intestinal GISTs expressed CD34, whereas all gastric, rectal, and oesophageal tumours were CD34 positive. All CD34 negative GISTs (20 cases) were located in the small intestine. All these 86 primary GISTs and their recurrent or metastatic tumours were completely negative for HHV-8 LNA- 1 .

\section{Clinical follow up}

After a mean follow up period of 30.2 months (median, 28.9; range, 6-112), 17 patients showed clinical malignancy. All primary tumours from these patients had a diameter of at least $5 \mathrm{~cm}$ (ranging from 5 to $27 \mathrm{~cm}$ ), and six had mitotic counts more than 5/50 HPF. The numbers of clinically malignant GISTs were: nine in the small bowel, seven in the stomach, and one in the rectum. These patients showed local recurrence, liver, lung, or regional nodal metastasis, or omental dissemination. According to the four tier system of predicting the aggressiveness of GISTs suggested by Fletcher et $a l,{ }^{4} 13$ primary lesions were of "high risk", four were of "intermediate risk", and none were of "low risk" or "very low risk." Both tumour size and mitotic activity were 
significantly strong predictors of clinical malignancy in univariate analysis ( $t$ test, $\mathrm{p}<0.005$ and $\mathrm{p}<0.05$, respectively).

\section{Molecular studies}

Both nested PCR and real time PCR showed that none of these 86 primary GISTs and their recurrent or metastatic tumours contained HHV-8 DNA, regardless of tumour status, namely: site derived from, tumour size, type of cellular components they contained, mitotic activity, CD34 positivity, primary or secondary tumour, history of other cancer, and clinical malignancy.

\section{DISCUSSION}

In 2002, Fletcher et al presented a scheme of risk assessment on the basis of tumour size and mitotic count. ${ }^{4}$ The scheme described four risk groups, namely: high risk, intermediate risk, low risk, and very low risk groups. This scheme was useful in predicting the malignant behaviour in our primary cases. Based on this scheme, 25 of our primary cases were high risk, 34 were intermediate risk, 24 were low risk, and three were very low risk tumours. ${ }^{4}$ None of our 17 malignant GISTs fell into the low risk and very low risk groups. In our series, the mean sizes of the malignant and benign GISTs were 13.6 and $5.7 \mathrm{~cm}$, respectively, and the mitotic counts of the malignant and benign tumours were 6.6 and 2.5/50 HPF, respectively. Increases in either primary tumour size or mitotic activity were significant predictors of tumour malignancy.

"Our results have demonstrated the lack of human herpesvirus type 8 infection in gastrointestinal stromal tumour cells, regardless of tumour status"

The CD34 expression rate of GISTs varies to some degree at different anatomical locations. Similar to that of previous studies, ${ }^{4518}$ the CD34 expression rate in our series was 77\% (66 of 86 tumours). Only 14 of the small intestinal GISTs studied here were CD34 positive, whereas those in the oesophagus, stomach, and rectum all expressed CD34.

HHV-8 is present in many disorders, including multicentric Castleman's disease, ${ }^{19}{ }^{20}$ primary effusion lymphoma, ${ }^{20}{ }^{21}$ some encephalitides, ${ }^{22}$ interstitial pneumonitis in human immunodeficiency virus infected patients, ${ }^{16}$ bone marrow biopsies of human immunodeficiency virus positive patients, ${ }^{23}$ bone marrow dendritic cells from multiple myeloma, ${ }^{24}$ reactive angioendotheliomatosis, ${ }^{25}$ oral plasmablastic lymphomas in patients with AIDS, ${ }^{26}$ and all types of KS. ${ }^{10}{ }^{11} \mathrm{KS}$ is similar to GIST in three respects at least, namely: (1) KS is most frequently found in the gastrointestinal tract, although cutaneous lesions do occur'; (2) KS is also composed of spindle or epithelioid cells, although

\section{Take home messages}

- Using immunohistochemistry, nested polymerase chain reaction $(P C R)$, and real time quantitative $P C R$, we found no evidence of human herpesvirus type 8 (HHV$8)$ infection in 86 gastrointestinal stromal tumours (GISTs)

- Thus, despite the similarities between Kaposi's sarcomas and GIST, HHV-8 does not appear to play a role in the pathogenesis of GIST, irrespective of tumour status erythrocytes can also be present ${ }^{7}$; and (3) KSs are nearly all positive for the CD34 antigen, ${ }^{910}$ similar to GISTs. ${ }^{18}$

The relation between HHV-8 and KS has been extensively studied and discussed. HHV-8 DNA has been consistently found in all types of KS, with or without an association with AIDS. ${ }^{10}{ }^{11}$ In particular, it was found in the CD34 positive spindle or epithelioid tumour cells of $\mathrm{KS}^{78}$ and has been implicated as an aetiological agent in this tumour. ${ }^{27}{ }^{28}$ Despite the similarities between GISTs and KSs, our results using immunostaining, nested PCR, and real time PCR have demonstrated the lack of HHV-8 infection in GIST tumour cells, regardless of tumour status. This strongly suggests that HHV-8 has no role to play in the pathogenesis of these tumours.

\section{ACKNOWLEDGEMENTS}

This study was supported by grant CMFHR9237 from the Chi-Mei Medical Centre, Tainan County, Taiwan. We thank Mr C-L Lu for his statistical assistance.

\section{Authors' affiliations}

C C Su, Department of Pathology, Buddhist Dalin Tzu Chi General Hospital, Dalin Town, Chiayi County 622, Taiwan

C F Li, Y L Liao, C N Lin, Department of Pathology, Chi-Mei Medical Centre, Tainan County 710, Taiwan

J J Lu, Division of Clinical Pathology, Department of Pathology, TriService General Hospital and National Defence Medical Centre, Taipei 114, Taiwan

\section{REFERENCES}

1 Miettinen M, El-Rifai W, H L Sobin L, et al. Evaluation of malignancy and prognosis of gastrointestinal stromal fumor: a review. Hum Pathol 2002;33:478-83.

2 Miettinen M, Sarlomo-Rikala M, Lasota J. Gastrointestinal stromal tumors: recent advances in understanding of their biology. Hum Pathol 1999;30:1213-18.

3 Miettinen M, Majidi M, Lasota J. Pathology and diagnostic criteria of gastrointestinal stromal tumors (GISTs): a review. Eur J Cancer 2002;38(suppl 5):S39-51

4 Fletcher CD, Berman JJ, Corless C, et al. Diagnosis of gastrointestinal stromal tumors: a consensus approach. Hum Pathol 2002;33:459-65.

5 Kontogianni K, Demonakou M, Kavantzas N, et al. Prognostic predictors of gastrointestinal stromal tumors: a multi-institutional analysis of 102 patients with definition of a prognostic index. Eur J Surg Oncol 2003;29:548-56.

6 Nichols CM, Flaitz CM, Hicks MJ. Treating Kaposi's lesions in the HIV-infected patient. J Am Dent Assoc 1993;124:78-84.

7 Roth WK, Brandstertter H, Sturzl M. Cellular and molecular features of HIVassociated Kaposi's sarcoma. AIDS 1992;6:895-913.

8 Gomez-Roman JJ, Ocejo-Vinyals JG, Sanchez-Velasco P, et al. Presence of human herpesvirus 8 DNA sequences in renal transplantation-associated pleural Kaposi sarcoma. Arch Pathol Lab Med 1999;123:1269-73.

9 Orenstein JM, Alkan S, Blauvelt A, et al. Visualization of human herpesvirus type 8 in Kaposi's sarcoma by light and transmission electron microscopy. AIDS 1997; 11:F35-45.

10 Buonaguro FM, Tornesello ML, Beth-Giraldo E, et al. Herpesvirus-like DNA sequences detected in endemic, classic, iatrogenic and epidemic Kaposi's sarcoma (KS) biopsies. Int J Cancer 1996;65:25-8.

11 Weiss RA, Whitby D, Talbot S, et al. Human herpesvirus type 8 and Kaposi's sarcoma. J Natl Cancer Inst Monogr 1998;23:51-4.

12 O'Leary J, Kennedy M, Howells D, et al. Cellular localisation of HHV-8 in Castleman's disease: is there a link with lymph node vascularity? Mol Pathol 2004;53:69-76.

13 Yang TY, Chen SC, Leach MW, et al. Transgenic expression of the chemokine receptor encoded by human herpesvirus 8 induces an angioproliferative disease resembling Kaposi's sarcoma. J Exp Med 2000;191:445-54.

14 Ryan P, Aarons S, Murray D, et al. Human herpesvirus 8 (HHV-8) detected in two patients with Kaposi's sarcoma-like pyogenic granuloma. J Clin Pathol 2002;55:619-22

15 Kubben FJ, Kroon FP, Hogendoorn PC, et al. Absence of Epstein-Barr virus (EBV) in a gastrointestinal stromal cell tumour (GIST) in an adult human immunodeficiency virus-seropositive patient with past EBV infection. Eur J Gastroenterol Hepatol 1997;9:721-4.

16 Muller A, Franzen C, Klussmann P, et al. Human herpesvirus type 8 in HIV infected patients with interstitial pneumonitis. J Infect 2000;40:242-7.

17 Stamey FR, Patel MM, Holloway BP, et al. Quantitative, fluorogenic probe PCR assay for detection of human herpesvirus 8 DNA in clinical specimens. J Clin Microbiol 2001;39:3537-40. 
18 Miettinen M, Sobin LH, Sarlomo-Rikala M. Immunohistochemical spectrum of GISTs at different sites and their differential diagnosis with reference to CD 117 (KIT). Mod Pathol 2000;13:1134-42.

19 Oksenhendler E, Boulanger E, Galicier L, et al. High incidence of Kaposi sarcoma-associated herpesvirus-related non-Hodgkin lymphoma in patients with HIV infection and multicentric Castleman disease. Blood 2002;99:2331-6.

20 Hengge UR, Ruzicka T, Tyring SK, et al. Update on Kaposi's sarcoma and other HHV8 associated diseases. Part 2: pathogenesis, Castleman's disease and pleural effusion lymphoma. Lancet Infect Dis 2002;2:344-52.

21 Cesarman E, Chang Y, Moore PS, et al. Kaposi's sarcoma-associated herpesvirus-like DNA sequences in AIDS-related body-cavity-based lymphomas. N Engl J Med 1995;332:1186-91.

22 Said JW, Tasaka T, de Vos S, et al. Kaposi's sarcoma-associated herpesvirus/ human herpesvirus type 8 encephalitis in HIV-positive and -negative individuals. AIDS 1997;11:1119-22.
23 Meggetto F, Cesarman E, Mourey L, et al. Detection and characterization of human herpesvirus-8-infected cells in bone marrow biopsies of human immunodeficiency virus-positive patients. Hum Pathol 2001;32:288-91.

24 Rettig MB, Ma HJ, Vescio RA, et al. Kaposi's sarcoma-associated herpesvirus infection of bone marrow dendritic cells from multiple myeloma patients. Science 1997;276:1851-4.

25 McMenamin ME, Fletcher CD. Reactive angioendotheliomatosis: a study of 15 cases demonstrating a wide clinicopathologic spectrum. Am J Surg Pathol 2002;26:685-97.

26 Cioc AM, Allen C, Kalmar JR, etal. Oral plasmablastic lymphomas in AIDS patients are associated with human herpesvirus 8. Am J Surg Pathol 2004;28:41-6.

27 Cathomas G. Kaposi's sarcoma-associated herpesvirus (KSHV)/human herpesvirus 8 (HHV-8) as a tumour virus. Herpes 2003;10:72-7.

28 Cheuk W, Wong KO, Wong CS, et al. Immunostaining for human herpesvirus 8 latent nuclear antigen-1 helps distinguish Kaposi sarcoma from its mimickers. Am J Clin Pathol 2004;121:335-42.

\section{$\mathrm{ECHO}$}

\section{PCR assay to the rescue in neurobrucellosis}

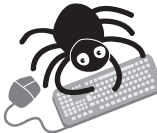

Please visit the Journal of Clinical Pathology website [www. jclinpath.com] for a link to the full text of this article.
$\mathrm{P}$ reliminary results suggesting that real time PCR assay may become the standard method for diagnosing neurological complications of brucellosis represent an important breakthrough for avoiding lasting damage to patients. It means that antibiotic treatment can be started within hours, rather than days or months it can take to reach a diagnosis by conventional means.

The other big advantage is the assay's closed, automated system, which limits the chances of cross contamination and makes it suitable for handling large numbers of specimens in a clinical laboratory-and without having to handle a class III pathogen directly.

Six patients with neurobrucellosis confirmed conventionally by a combination of clinical signs and symptoms and isolation of Brucella abortus or detection of specific agglutinins, or both, all showed specific B abortus sequences in CSF with the assay. Only four samples (66\%) were positive by seroagglutination and two (33\%) by direct culture. Symptoms had lasted on average 90 (range 5-365) days before diagnosis. All patients were Spanish: only one had no obvious exposure to $B$ abortus.

Each CSF sample was tested for $B$ abortus by culturing on isolation media, by testing for specific antibodies and testing for $B$ abortus DNA by LightCycler real time PCR assay.

Brucellosis is endemic around the Mediterranean, in India, Mexico, Central and South America. Almost $20-40 \%$ of infections cause complications, albeit only $1-2 \%$ in the CNS, but these can be serious. Low antibiotic titres in the CSF and the low yield of culture make diagnosis difficult.

A Colmenero JD, et al. Journal of Neurology, Neurosurgery, and Psychiatry 2005;76:1025-1027. 